“( 2014 IEEE. Personal use of this material is permitted. Permission from IEEE must be obtained for all other uses, in any current or future media, including

reprinting/republishing this material for advertising or promotional purposes, creating new collective works, for resale or redistribution to servers or lists, or reuse of any copyrighted component of this work in other works." 


\title{
Enhancing Accuracy of Mental Fatigue Classification using Advanced Computational Intelligence in Electroencephalography System
}

\author{
Rifai Chai, Member, IEEE, Yvonne Tran, Ashley Craig, Sai Ho Ling, Senior Member, IEEE and \\ Hung T. Nguyen, Senior Member, IEEE
}

\begin{abstract}
A system using electroencephalography (EEG) signals could enhance the detection of mental fatigue while driving a vehicle. This paper examines the classification between fatigue and alert states using an autoregressive (AR) model-based power spectral density (PSD) as the features extraction method and fuzzy particle swarm optimization with cross mutated of artificial neural network (FPSOCM-ANN) as the classification method. Using 32-EEG channels, results indicated an improved overall specificity from $76.99 \%$ to $\mathbf{8 2 . 0 2 \%}$, an improved sensitivity from $\mathbf{7 4 . 9 2}$ to $\mathbf{7 8 . 9 9 \%}$ and an improved accuracy from $75.95 \%$ to $80.51 \%$ when compared to previous studies. The classification using fewer EEG channels, with eleven frontal sites resulted in $77.52 \%$ for specificity, $\mathbf{7 3 . 7 8 \%}$ for sensitivity and $\mathbf{7 5 . 6 5 \%}$ accuracy being achieved. For ergonomic reasons, the configuration with fewer EEG channels will enhance capacity to monitor fatigue as there is less set-up time required.
\end{abstract}

\section{INTRODUCTION}

Fatigue whilst driving is known to be as major cause of accidents in transportation. Fatigue symptoms include increased feelings of tiredness and reduced alertness that results in decreased reaction time to respond to changes in conditions and to perform a task. Fatigue is known to lead to driving related traffic accidents, injuries and fatalities [1]. As a result, there is a need for an automatic detection and monitoring of driver related fatigue.

From the physiological parameters point of view, a counter-fatigue monitoring device can use the signals from eye movement using electrooculography (EOG) [2], heart rate variability (HRV) using electrocardiography (ECG) [3] and brain signal using electroencephalography (EEG) $[4,5]$. This paper explores fatigue detection using parameters from EEG signals to form a brain monitoring device. A system using EEG is believed to be effective in detecting mental fatigue and provide an output that could alert a driver, thus preventing attention lapses that may cause a traffic accident. The EEG-based system of this application would enhance the natural central nervous system output.

Rifai Chai, Sai Ho Ling, and Hung T. Nguyen are with Key Centre for Health Technologies, Faculty of Engineering and Information Technology, University of Technology, Sydney, Broadway NSW 2007, Australia. E-mail contacts: Rifai.Chai@student.uts.edu.au, Steve.Ling@uts.edu.au and Hung.Nguyen@uts.edu.au.

Yvonne Tran is with Key Centre for Health Technologies University of Technology, Sydney and Rehabilitation Studies Unit, University of Sydney. Email contact: Yvonne.Tran@uts.edu.au.

Ashley Craig is with the Rehabilitation Studies Units, University of Sydney. Email contact: a.craig@sydney.edu.au
The functional basic components of EEG-based system classification consists of several elements including signal measurement using the EEG and computational intelligence of the EEG signal which includes signal pre-processing, features extraction and classification processes with the output the classifier [6, 7]. For the features extraction in the EEG analysis, power spectral density (PSD) has been used widely in the EEG analysis especially in fatigue study [8, 9]. The power spectrum estimation is calculated from the Fast Fourier Transform (FFT) which converts the time based EEG data into the following frequency bands of EEG rhythms: delta $(\delta)$, theta $(\theta)$, alpha $(\alpha)$ and beta $(\beta)$. For the classifier in the EEG study, two groups are considered; linear and nonlinear classifiers. A linear classifier is simpler than a nonlinear classifier, as it uses a linear mathematical model only for the classifier training, however, for EEG-based classification, it will be a less effective method. As a result, a non-linear method of classification should be investigated given they could lead to improve classification of fatigue. For instance, an artificial neural network (ANN) is a popular nonlinear classifier algorithm used in biomedical study [10].

An experimental study was conducted using the EEG to measure mental fatigue versus alert brain activity states to determine potential as a fatigue countermeasure device. The overall EEG fatigue classification was found to be around $76 \%$ on the original dataset without any of the regrouping methods for the dataset [8]. The PSD was used as the feature extraction method. The ANN was used for the classification algorithm.

To continue this line of research, this paper explores the use of advanced computational intelligence techniques in order to improve the accuracy of the fatigue versus alert classification. An alternative method based on the autoregressive (AR) modelling of PSD estimation is used as the feature extraction method. For the classifier, a new optimization technique in computational intelligence namely a fuzzy particle swarm optimization with cross mutated (FPSOCM) has been introduced [11]. Recently, the FPSOCM has been applied to optimize the training of an artificial neural network (FPSOCM-ANN) for three mental task classifications (mental arithmetic, mental letter composing and imagining a Rubik's cube rolling) $[12,13]$ with a promising result of improved accuracy. Thus, the FPSOCM-ANN is examined as the classification algorithm in this study for the fatigue vs. alert states classification. Also for ergonomic reasons, a classification with fewer EEG channels was also included. Previous results have shown that there was increased fast wave activity of fatigue mostly in the 
frontal area [5]. This paper also includes the classification using only the frontal sites of the EEG channels.

\section{Methodology}

\section{A. Components for EEG-based Fatigue Classification}

The process began with the collection of brain signals using a multi-channel EEG system for data acquisition during a fatigue study with non-professional drivers. The output of the data acquisition process is in a digital form which will be continued with the features extraction that transforms the signals into useful features corresponding to the mental strategy of the system. In this study the mental strategy is for fatigue detection. As a result, output of the classification forms two states, fatigue and alert mental status.

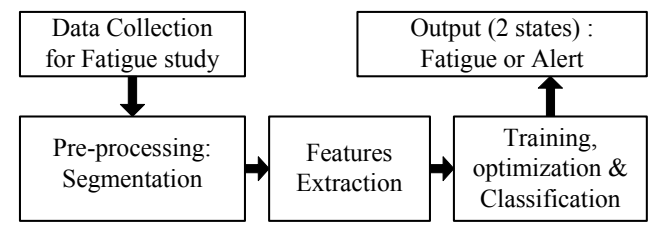

Figure 1. Components for EEG-based fatigue classification

\section{B. Procedure for Data Collection of Fatigue Study}

Data set of fourteen healthy participants with the age between 18 and 55 years obtained from the previous study was used [8]. Participants were given information of the study and the informed consent form was signed before commencing the experiment. The study was approved by the institutional research ethics committee. The Divided Attention Steering Simulator (DASS) from Stowood Scientific Instruments was used for a driving simulator task. Here, participants needed to ensure that they kept driving at the centre of the road in the simulation task until the signs of fatigue were shown. The experiment was stopped if facial signs were detected (such as nodding and extended eyes closure) or if they were driving off the road in the simulation driving task for greater than 15 seconds. The maximum time for the simulated driving was specified at $2 \mathrm{hrs}$.

EEG signals were recorded by attaching a 32 channels EEG system, the Active-Two system from Biosemi with the electrode position based on the International 10-20 system. These positions are: FP1, AF3, F7, F3, FC1, FC5, T7, C3, CP1, CP5, P7, P3, PZ, PO3, O1, OZ, O2, PO4, P4, P8, CP6, CP2, C4, T8, FC6, FC2, F4, F8, AF4, FP2, FZ and CZ. The sampling rate of the system was down sampled from $2048 \mathrm{~Hz}$ to $256 \mathrm{~Hz}$.

\section{Signal Pre-processing}

A program using the second-order blind identification (SOBI) and canonical correlation was used for removing artifact [14]. For the alert group of data, the first 5 mins of EEG data when starting the driving simulation task was chosen and the fatigue group of data was selected from the last 5 mins of EEG data before the task was stopped. Then in each group of the data (alert and fatigue), 20s duration of segment was taken and the first 20s segment was selected for further analysis that had the least movement artifact. As a result, 20s of alert state and another 20s of fatigue state were available for each participant. A moving window of $2 \mathrm{~s}$ with overlapping $0.25 \mathrm{~s}$ was applied to the $20 \mathrm{~s}$ segments which provided 73 overlapping segments on each state. With the 14 participants, a total of 1022 units of datasets were formed on alert state and another 1022 units for the fatigue state.

\section{Features Extraction}

An autoregressive (AR) model was applied for calculating the power spectrum. The calculation of the AR modelling is as follows:

$$
x(m)=\sum_{k=1}^{P} a(k) x(m-k)+e(m)
$$

where $x(m)$ represents the signal at time $m, P$ is the order of the AR, $a(k)$ represents the AR coefficients and $e(m)$ represents the white noise with the zero means error and finite variance. It is assumed to be a random process which is independent of the previous value of the signal. The Burg method is one of the AR spectral estimation methods which have been used in the EEG application [9]. The PSD from the calculation of the Burg method ( $\left.\hat{P}_{B U R G}(\omega)\right)$ is as follows:

$$
\hat{P}_{B U R G}(\omega)=\frac{\hat{e}_{p}}{\left|1+\sum_{k=1}^{P} \hat{a}_{p}(k) e^{-j k \omega}\right|^{2}}
$$

where $\hat{a}_{p}(k)$ are the estimated AR parameters and $\hat{e}_{p}$ are the reflection coefficients which are to obtain the total least square error. The AR order of 25 was used in this study.

AR-based PSD is applied to 2s of each EEG segment with 512 points for $256 \mathrm{~Hz}$ of sampling rate. This converts the time EEG segment into the frequency domain. There are 4 EEG bands used for the features which include: $\delta(0.5-3 \mathrm{~Hz})$, $\theta(3.5-7.5 \mathrm{~Hz}), \alpha(8-13 \mathrm{~Hz})$ and $\beta(13.5-30 \mathrm{~Hz})$. The total power of each EEG band was calculated using the trapezoidal rule and this provided 4 power values for each of the EEG bands. A total of 128 units of power (32 EEG channels) or 44 units of power (frontal sites of EEG channels) were made available as the input features on each set of segment which were used for the classification process.

\section{E. Classification}

The fuzzy particle swarm with cross mutated operation of artificial neural network (FPSOCM-ANN) as a non-linear method was used for the classifier. It is composed of a three layers feed forward neural network with a single hidden layer as shown in Fig. 2. The FPSOCM was used to optimize the neural network training.

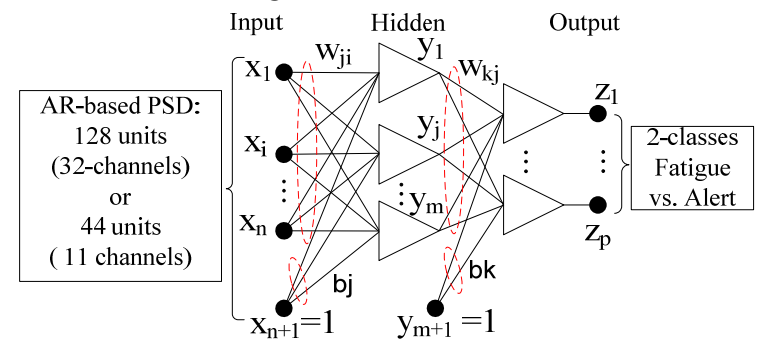

Figure 2. ANN structure for the fatigue vs. alert classification 
These features were normalized into [0 1$]$ range prior to the classification as the log-sigmoid was used for the transfer function. The output vector $z_{k}$ and the normalization are computed as follows:

$$
\begin{aligned}
z_{k}(x, w) & =f\left(b_{k}+\sum_{j=1}^{l} w_{k j} f\left(b_{j}+\sum_{i=1}^{m} w_{j i} x_{i}\right)\right) \\
X_{i} & =\left(X-X_{\text {min }}\right) /\left(X_{\max }-X_{\text {min }}\right)
\end{aligned}
$$

where $f($.) is the transfer functions of ANN, $m$ is the input nodes number $(i=1,2, \ldots, m), l$ is the hidden nodes number $(j=1,2, \ldots, l), p$ is the number of output $(k=1,2, \ldots, p), w_{j i}$ is the weight to the hidden unit $y_{j}$ from input unit $x_{i}, w_{k j}$ is the weights to output $\left(z_{k}\right)$ from hidden unit $\left(y_{j}\right), b_{j}$ and $b_{k}$ are the biases. $X_{i}$ is the result of features after normalization. $X$ is the result of features before normalization. $X_{\min }$ is the minimum value features and $X_{\max }$ is the maximum value of the features.

In the FPSOCM [11] for ANN optimization, a fuzzy inertia weight and a cross-mutated operation were introduced for performance searching improvement and to handle the issue of local minimal trapping. The process was started by the initialization of the particle swarm. The particle swarm $X(t)$ was constructed from the ANN parameters and was evaluated by fitness function. Prior to the iterative PSO process, the probability of cross mutated (CM) operation was defined. The value of inertia weight was controlled by two inputs of the fuzzy inference system (FIS), the normalized standard deviation (SD) of cost value among all the particles and the iteration stage. After the inertia weight has been calculated, the velocity was updated.

The FIS was used to find the control parameter. The velocity of all particle element swarm was evaluated by defined probability of $\mathrm{CM}$ during the $\mathrm{CM}$ process. A random particle with the value in the range $\left[\begin{array}{ll}0 & 1\end{array}\right]$ was generated. If the random particle value has a value more than the probability of the CM operation, then the $\mathrm{CM}$ operation will be calculated. The maximum velocity value was used as the limit. An updated particle swarm was generated after the CM operation process. The process was repeated until a defined number of iterations $(T)$ was reached. The aim for the FPSOCM-ANN is to minimize the fitness value. Fig. 3 shows the FPSOCM procedure.

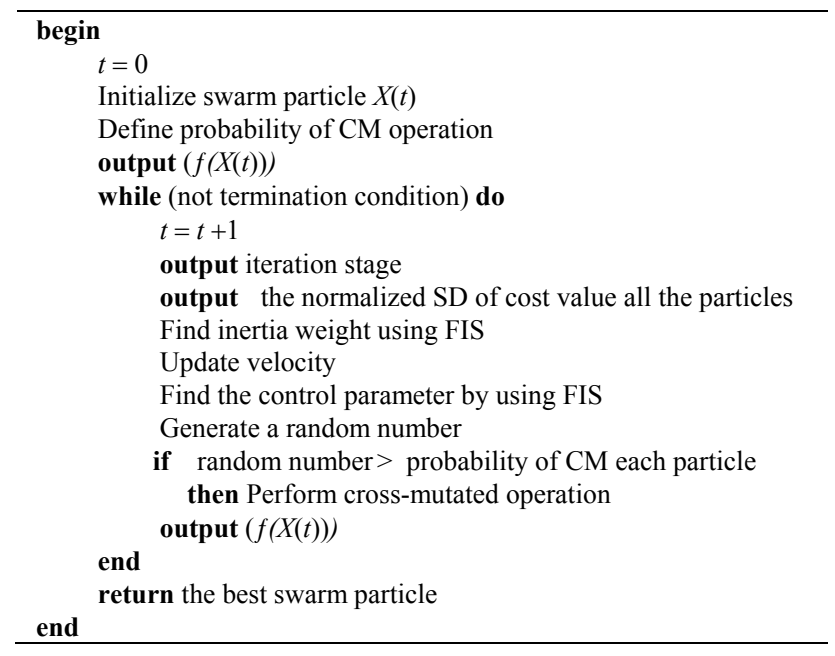

Figure 3. The procedure for FPSOCM-ANN
The parameters of training of the FPSOCM-ANN for fatigue vs. alert classification were as follows: the size of the swarm was 50 , acceleration constant was 2.05 , the value of maximum velocity was 0.2 , each CM probability was 0.005 and the iteration number was 2000. The training of the FPSOCM-ANN was repeated ten times for different hidden neurons with variation from 3-25 units to obtain the best number with highest classification accuracy. As a result the accuracies were the mean value of ten accuracy results.

A $k$-fold cross-validation ( $k=3$ ) was used for the accuracy performance evaluation to avoid the issue of over-fitting in machine learning. The total of 1022 dataset from fatigue was divided into 3 folds (fold $1=336$ sets, fold $2=336$ sets and fold $3=350$ sets). One of these folds (hold out set) was used for the testing. The remaining folds were used to train the classifier function. The mean value accuracy of the three folds was used for the overall accuracy.

\section{RESULtS}

The results of the classification between fatigue and alert states using the 32 EEG channels are shown in Table 1. For the first fold, the correctly classified rate for the alert state was $80 \%$, for the fatigue state was $78.57 \%$ and the accuracy was $78.57 \%$. For the second fold, the correctly classified rate for the alert state was $82.74 \%$, for the fatigue state was $80.65 \%$ and the accuracy was $81.69 \%$. In the third fold, the correctly classified rate for the alert state was $83.33 \%$, for the fatigue state was $79.17 \%$ and the accuracy was $81.25 \%$. The mean of the classification rate of the three folds for the alert state (specificity) was $82.02 \%$. The mean of the classification rate of the three folds for the fatigue state (sensitivity) was $82.02 \%$. The overall accuracy of the classification fatigue vs. alert states was $80.51 \%$.

TABLE I. RESULT CLASSIFICATION OF FATIGUE VS. ALERT OF 32 EEG

\begin{tabular}{|c|c|c|c|c|}
\hline K-Folds & & Alert & Fatigue & Overall \\
\hline \multirow{3}{*}{$\begin{array}{l}1 \text { st- } \\
\text { Fold }\end{array}$} & Total test data & 350 & 350 & 700 \\
\hline & Correctly classified & 280 & 270 & 550 \\
\hline & Classification Rate & $80 \%$ & $77.14 \%$ & $78.57 \%$ \\
\hline \multirow{3}{*}{$\begin{array}{l}\text { 2nd- } \\
\text { Fold }\end{array}$} & Total test data & 336 & 336 & 672 \\
\hline & Correctly classified & 278 & 271 & 549 \\
\hline & Classification Rate & $82.74 \%$ & $80.65 \%$ & $81.69 \%$ \\
\hline \multirow{3}{*}{$\begin{array}{l}\text { 3rd- } \\
\text { Fold }\end{array}$} & Total test data & 336 & 336 & 672 \\
\hline & Correctly classified & 280 & 266 & 546 \\
\hline & Classification Rate & $83.33 \%$ & $79.17 \%$ & $81.25 \%$ \\
\hline \multicolumn{2}{|c|}{ Mean of Classification Rate } & $82.02 \%$ & $78.99 \%$ & $80.51 \%$ \\
\hline & & Specificity & Sensitivity & Accuracy \\
\hline
\end{tabular}
CHANNELS WITH 3-FOLD CROSS VALIDATION

The comparison result to the previous study [8] is shown in Table II. The original result from the previous study was the one without any re-grouping dataset methods. In the previous study the overall specificity was $76.99 \%$ and this value is improved in this paper to $82.02 \%$. The overall sensitivity is also increased; the sensitivity of the previous study was $74.92 \%$ and this paper provides improved sensitivity to $78.99 \%$. The overall accuracy of the classification between fatigue and alert states is improved to $80.51 \%$ in this paper from $75.96 \%$ of the previous study. 
TABLE II. COMPARISON RESULT TO THE PREVIOUS STUDY ON THE SAME DATASET OF 32 EEG CHANNELS.

\begin{tabular}{|c|c|c|c|}
\hline \hline \multirow{3}{*}{ Study } & \multicolumn{2}{|c|}{ Correctly identified } & \multirow{2}{*}{$\begin{array}{c}\text { Accuracy } \\
\text { (\%) }\end{array}$} \\
\cline { 2 - 3 } & Alert & Fatigue & \\
\cline { 2 - 3 } & Specificity (\%) & Sensitivity (\%) & \\
\hline Previous study [8] & $76.99 \%$ & $74.92 \%$ & $75.96 \%$ \\
\hline This Study & $82.02 \%$ & $78.99 \%$ & $80.51 \%$ \\
\hline \hline
\end{tabular}

TABLE III. RESULT CLASSIFICATION OF FATIGUE VS. ALERT OF FRONTAL EEG CHANNELS (11 CHANNELS) WITH 3-FOLD CROSS VALIDATION

\begin{tabular}{|c|c|c|c|c|}
\hline "K-Folds & & Alert & Fatigue & Overall \\
\hline \multirow{3}{*}{$\begin{array}{l}1 \text { st- } \\
\text { Fold }\end{array}$} & Total test data & 350 & 350 & 700 \\
\hline & Correctly classified & 264 & 258 & 522 \\
\hline & Classification Rate & $75.43 \%$ & $73.71 \%$ & $74.57 \%$ \\
\hline \multirow{3}{*}{$\begin{array}{l}\text { 2nd- } \\
\text { Fold }\end{array}$} & Total test data & 336 & 336 & 672 \\
\hline & Correctly classified & 258 & 245 & 503 \\
\hline & Classification Rate & $76.79 \%$ & $72.92 \%$ & $74.85 \%$ \\
\hline \multirow{3}{*}{$\begin{array}{l}\text { 3rd- } \\
\text { Fold }\end{array}$} & Total test data & 336 & 336 & 672 \\
\hline & Correctly classified & 270 & 251 & 521 \\
\hline & Classification Rate & $80.30 \%$ & 74.70 & $77.53 \%$ \\
\hline \multicolumn{2}{|c|}{ Mean of Classification Rate } & $77.52 \%$ & 73.78 & $75.65 \%$ \\
\hline & & Specificity & Sensitivity & Accuracy \\
\hline
\end{tabular}

For improved ergonomic operation, a system with fewer EEG channels is preferable as it requires less time for set-up. The result from the previous study reported that there was an increase of fast wave activity of fatigue mostly in the frontal area [5]. As a result, this paper investigates the classification on the frontal sites with a total of 11 EEG channels (FP1, AF3, F7, F3, FC1, FC2, F4, F8, AF4, FP2 and FZ).The results of the classification between fatigue and alert states using the frontal sites of 11 EEG channels are shown in Table III. For the first fold, the correctly classified rate for the alert state was $75.43 \%$, for the fatigue state was $73.71 \%$ and the accuracy was $74.57 \%$. For the second fold, the correctly classified rate for the alert state was $76.79 \%$, for the fatigue state was $72.92 \%$ and the accuracy was $74.85 \%$. In the third fold, the correctly classified rate for the alert state was $80.30 \%$, for the fatigue state was $74.70 \%$ and the accuracy was $77.53 \%$. The mean of the classification rate of the three folds for the alert state (specificity) was $77.52 \%$. The mean of the classification rate of the three folds for the fatigue state (sensitivity) was $73.78 \%$. The overall accuracy of the classification fatigue vs. alert states was $75.65 \%$.

\section{CONCLUSION}

In this paper advanced computational intelligence was applied for fatigue versus alert classification using the ARbased PSD as the feature extraction algorithm and FPSOCM-ANN as the classification algorithm. The dataset was taken from an experimental study that obtained the fatigue and alert states using an EEG system attached on the scalp [8]. The results for 32-EEG channels showed an improved specificity from $76.99 \%$ of the previous study to $82.02 \%$ in this paper, an improved sensitivity from $74.92 \%$ of the previous study to $78.99 \%$ in this paper and an improved accuracy from $75.96 \%$ of the previous study to $80.51 \%$ in this paper. The resultant classification using fewer EEG channels of only frontal sites (11 channels) of EEG channels has also been investigated with the overall specificity at $77.52 \%$, sensitivity at $73.78 \%$ and accuracy at $75.65 \%$. The benefit of using fewer EEG channels include less set-up time required and this may assist in an eventual fatigue real-time monitoring device based on EEG signals.

\section{ACKNOWLEDGMENT}

The authors would like to thank Dr Nirupama Wijesuriya for her contribution to the work for collecting the data in this study.

\section{REFERENCES}

[1] W. Vanlaar, H. Simpson, D. Mayhew, and R. Robertson, "Fatigued and drowsy driving: A survey of attitudes, opinions and behaviors," Journal of Safety Research, vol. 39, pp. 303-309, 2008.

[2] S. Hu and G. Zheng, "Driver drowsiness detection with eyelid related parameters by Support Vector Machine," Expert Systems with Applications, vol. 36, pp. 7651-7658, 2009.

[3] Y. Tran, N. Wijesuriya, M. Tarvainen, P. Karjalainen, and A. Craig, "The relationship between spectral changes in heart rate variability and fatigue," Journal of Psychophysiology, vol. 23, pp. 143-151, 2009.

[4] A. Craig, Y. Tran, N. Wijesuriya, and P. Boord, "A controlled investigation into the psychological determinants of fatigue," Biological Psychology, vol. 72, pp. 78-87, 2006.

[5] A. Craig, Y. Tran, N. Wijesuriya, and H. Nguyen, "Regional brain wave activity changes associated with fatigue," Psychophysiology, vol. 49, pp. 574-582, 2012.

[6] J. R. Wolpaw and E. W. Wolpaw, "Brain-computer interfaces: something new under the sun," in Brain-Computer Interfaces: Principles and Practice, J. R. Wolpaw and E. W. Wolpaw, Eds. New York: Oxford University Press, 2012, pp. 3-12.

[7] B. He, S. Gao, H. Yuan, and J. Wolpaw, "Brain-Computer Interfaces," in Neural Engineering, B. He, Ed.: Springer US, 2013, pp. 87-151.

[8] Y. Tran, A. Craig, N. Wijesuriya, and H. Nguyen, "Improving classification rates for use in fatigue countermeasure devices using brain activity," in Proc. of the 32 Annual International Conference of the IEEE Engineering in Medicine and Biology Society (EMBC), 2010, pp. 4460-4463.

[9] O. Faust, R. U. Acharya, A. R. Allen, and C. M. Lin, "Analysis of EEG signals during epileptic and alcoholic states using AR modeling techniques," IRBM, vol. 29, pp. 44-52, 2008.

[10] H. T. Nguyen, "Intelligent technologies for real-time biomedical engineering applications," Int. J. Automation and Control, vol. 2, Nos.2/3, pp. 274-285, 2008.

[11] S. H. Ling, H. T. Nguyen, F. H. F. Leung, K. Y. Chan, and F. Jiang, "Intelligent fuzzy particle swarm optimization with cross-mutated operation," in Proc. 2012 IEEE Congress on Evolutionary Computation (CEC), 2012, pp. 3009-3016.

[12] R. Chai, S. H. Ling, G. P. Hunter, Y. Tran, and H. T. Nguyen, "Classification of Wheelchair Commands using Brain Computer Interface: Comparison between Able-Bodied Persons and Patients with Tetraplegia " in Proc. of the 35 Annual International Conference of the IEEE Engineering in Medicine and Biology Society (EMBC) 2013, Osaka, 2013, pp. 989-992.

[13] R. Chai, S. Ling, G. Hunter, Y. Tran, and H. Nguyen, "Brain Computer Interface Classifier for Wheelchair Commands using Neural Network with Fuzzy Particle Swarm Optimization," IEEE Journal of Biomedical and Health Informatics, 2013(In Press).

[14] Y. Tran, R. A. Thuraisingham, A. Craig, and H. Nguyen, "Evaluating the efficacy of an automated procedure for EEG artifact removal," in Proc. of the 31 Annual International Conference of the IEEE Engineering in Medicine and Biology Society (EMBC), 2009, pp. 376-379. 